\title{
SORPTION ISOTHERMS FOR FOOD PRODUCTS: STUDY OF MODELS AGREEMENT
}

\author{
FABIANO RIBEIRO NASCIMENTO* \\ JEFFERSON LUIZ GOMES CÔRREA** \\ SORAIA VILELA BORGES*** \\ POLIANA GASPAR TOSATO****
}

\begin{abstract}
The knowledge of sorption isotherms is important for establishing conditions of storage and of processes like drying. There are several models for fitting sorption isotherms. This work presents a study about the agreement of 40 mathematical models of sorption isotherms to experimental data of 53 food products. The quadratic residual sum and the standard error were the criteria of evaluation. For the major part of the products, the best agreement was obtained with equation of Jaafar and Michalowski, if temperature or saturation pressure were not considered as a variable. For cases where temperature or saturation pressure were considered, the equation of Strohman and Yoerger was the one with the best agreement for most of the products. Ross equation, based on thermodynamics aspects, was also tested for some products, but the agreement was just satisfactory.
\end{abstract}

KEY-WORDS: FOOD -STORAGE, DRYING; THERMODYNAMICS; SORPTION ISOTHERMS.

* Engenheiro de Alimentos, formado pela Universidade Federal de Lavras (UFLA), Lavras, MG (e-mail: fabianor@ymail.com).

** Engenheiro Químico, Professor, Doutor em Engenharia Mecânica, UFLA, Lavras, MG (e-mail: jefferson@ dca.ufla.br).

*** Engenheira Química, Professora, Doutora em Engenharia de Alimentos, UFLA, Lavras, MG (e-mail: sborges@dca.ufla.br).

**** Engenheira de Alimentos, mestranda em Ciência dos Alimentos, Departamento de Ciência dos Alimentos, UFLA, Lavras, MG (e-mail: pgtosato@hotmail.com). 


\section{INTRODUCTION}

Water represents the compound of biological materials of higher percentage in most of food products. It is a vehicle to biological, chemical and enzymatic reactions. It also influences sensorial properties. Moisture content of a product in equilibrium with the boundary atmosphere is called equilibrium moisture content. It is function of relative humidity, air temperature and interactions between water and solid material. The relationship between moisture content and its water activity (or relative humidity) in a certain temperature is called sorption isotherm.

Sorption isotherms are important, among other factors, to establish storage conditions of certain food and to report adsorptions properties of food products (DU, ZHOU and YANG, 2007; ASSUNÇÃO and PENA, 2007; YANG et al., 2009). Iglesias and Chirife (1982) published a study of agreement of equations of sorption isotherms of several food products. Those authors worked only with models of two parameters and noted that there is not an universal equation that fits all the tested food experimental isotherm data. The differences among the isotherm behavior for different products result, among other factors, from the relationship between moisture content and the structure of the food. Basu, Shivhare \& Mujumdar (2006) in a review of sorption isotherms presented a great discussion about thermodynamic aspect, determination methods, with 19 mathematical models and 12 products. Those authors verified that GAB model (VAN DEN BERG, 1983 ) was the one with best agreement for most of the food products. The GAB (VAN DEN BERG, 1983) and BET (BRUNAUER, EMMETT \& TELLER., 1938) equations are commonly used for food products (GOULA et al., 2008; VEGA-GALVÉZ et al., 2009; FARAHNAKY, ANSARI \& MAJZOOBI, 2009; FABRA et al., 2009; THYS et al., 2010; SYAMALADEVI et al., 2010; KOC et al., 2010). Limousin et al. (2007) observed that the choice for a model should be based on the simplest model, with physical argument. Roman et al. (2004) recommended the use of an equation based on the components of the food, the Ross one.

The goal of this work was the obtainment of agreement of mathematical models for food products experimental data of isotherms. Experimental data were obtained in the literature. Such data were extracted only from tables. Data from graphics were not used here, for a greater reliance. Until the present moment, there is not a work with the great number of equations and products reported in this study.

\section{MATERIAL AND METHODS}

\subsection{MATERIALS}

The food products tested in the present work were the following ones: acacia, açai, alginic acid, amaranth seeds, apple, apricot, banana, barley, black bean seed, buckwheat, coffee cherry, beneficed coffee, coffee products, colza, corn, cotton seed, cowpea, cupuaçu, dry beans, soy bran, flaxseed, flour, gluten, grape, kiwi, lean beef, maize starch, mushroom, oats, peanuts, pear, pork muscle, potato, potato starch, pulp of West Indian cherry, quinoa grains, rice (milled, rough and whole grain), rye, shelled corn, shelled popcorn, sorghum, soybeans, pink shrimp, starch, sugar beet seeds, tragacanth and wheat.

\subsection{MATHEMATICAL MODELS}

The mathematical models used are presented in Table 1.

\subsection{METHODOLOGY}

The equations used and its references are presented in Table 1. These equations were adjusted with a non-linear estimation method and quasi-Newton approach with convergence criterion of $1.00 \times 10^{-4}$. Initial value of 0.10 and initial estimative of 0.50 were used for all the estimated parameters. The agreements were selected based on determination coefficient $\left(r^{2}\right)$ and estimative standard error (SE) given by equations 41 and 42, respectively, as recommended and used by Basu, Shivhare \& 
Mujumdar (2006). Only values of $r^{2}$ higher than 0.99 were considered for equations that do not take temperature and/or vapor pressure of saturation into account. The best agreement was the criterion for equations that consider at least one of these variables (temperature or vapor pressure):

$$
\begin{gathered}
r^{2}=\frac{\sum_{1}^{n}(P R E D-O B S)^{2}}{\sum_{1}^{n}(O B S-\overline{O B S})^{2}} \\
S E=\sqrt{\frac{\sum_{1}^{n}(O B S-P R E D)^{2}}{n}}
\end{gathered}
$$

Where: $\mathrm{OBS}=$ observed value; $\overline{\mathrm{OBS}}=$ the average value of observed values; $\mathrm{PRED}=$ the predict value; and $\mathrm{n}=$ the number of observations.

\begin{tabular}{|c|c|c|c|c|c|}
\hline Equation & Ref & $\mathrm{N}^{\circ}$ & Equation & $\operatorname{Ref}^{\circ}$ & $\mathrm{N}^{\circ}$ \\
\hline$\varphi=\frac{X / s_{1}}{s_{2}\left(1-X / s_{1}\right)}$ & 38 & 1 & $\varphi=\frac{\mathrm{s}_{1}}{\exp \left(\mathrm{s}_{2} \mathrm{X}^{-\mathrm{S}_{3}}\right)}$ & 59 & 21 \\
\hline$X=\frac{s_{1} s_{2}}{(1-\varphi)\left[1+\left(s_{2}-1\right) \varphi\right]}$ & 9 & 2 & $h(1-\varphi)=-s_{1} T+s_{2} x^{s_{3}}$ & 45 & 22 \\
\hline $\log (\log (100 \varphi))=s_{2} \log \left(\frac{X}{s_{1}}\right)$ & 24 & 3 & $\varphi=s_{3}\left(1-\exp \left(-s_{1} \mathbb{X}^{s_{2}}\right)\right.$ & 45 & 23 \\
\hline$X=\frac{s_{1}}{\ln (\varphi)}+s_{2}$ & 37 & 4 & $\varphi=1-\exp \left[-\mathrm{s}_{1}\left(\mathrm{~s}_{2}+\mathrm{T}\right) \mathrm{X}^{\mathrm{s}_{3}}\right]$ & 41 & 24 \\
\hline$h(\varphi)=s_{1}-\frac{s_{2}}{X^{2}}$ & 25,26 & 5 & $\varphi=\exp \left[-X^{s_{3}} \exp \left(s_{1}-s_{2} T\right)\right]$ & 41 & 25 \\
\hline$X=\frac{s_{1} s_{2} \varphi}{1+\varphi S_{2}}(1+\varphi)$ & 30 & 6 & $\varphi=\exp \left[-\frac{s_{1}}{s_{3}+T} \exp \left(-s_{2} X\right)\right]$ & 41 & 26 \\
\hline$h(h(1 / \varphi))=h s_{1}+X h s_{2}$ & 7 & 7 & $\mathbf{h}(1-\varphi)=-\mathrm{s}_{1} \mathrm{X}^{\mathrm{s}_{2}} \mathrm{~T}^{\mathrm{s}_{3}}$ & 41 & 27 \\
\hline $\mathbf{h}(1-\varphi)=-\mathrm{s}_{1} \mathrm{X}^{\mathrm{s}_{2}}$ & 29 & 8 & $X=\left(-s_{2} T+s_{3}\right) \frac{s_{1} \varphi}{(1-\varphi)\left[1+\left(s_{1}-1\right) \varphi\right]}$ & 43 & 28 \\
\hline$h(X)=\ln \left(s_{1}\right)-s_{2} \varphi$ & 12 & 9 & $\varphi=\exp \left[-\mathrm{s}_{1} T^{\mathrm{s}_{2}} \exp \left(-\mathrm{s}_{3} \mathrm{~T}^{\mathrm{s}_{4}} \mathrm{X}\right)\right.$ & 14 & 29 \\
\hline
\end{tabular}

TABLE 1 - MATHEMATICAL MODELS OF SORPTION ISOTHERMS 


\begin{tabular}{|c|c|c|c|c|c|}
\hline Equation & Ref & $\mathrm{N}^{\circ}$ & Equation & $\operatorname{Ref}^{\circ}$ & $\mathrm{N}^{\circ}$ \\
\hline$h(\varphi)=-\frac{s_{1}}{\mathbb{R}} \exp \left(-s_{2} X\right)$ & 15 & 10 & $1-\varphi=\exp \left[-\mathrm{s}_{1} \mathrm{~T}^{\mathrm{s}_{2}} \mathrm{X}^{\left.\mathrm{s}_{3} \mathrm{~T}^{\mathrm{s}_{4}}\right]}\right]$ & 17 & 30 \\
\hline$\varphi=\frac{s_{1}+X}{s_{2}+X}$ & 42 & 11 & $h \varphi=s_{1} h P_{s}(T) \exp \left(s_{2} X\right)+s_{3} \exp \left(s_{4} X\right)$ & 54 & 31 \\
\hline$X=s_{1}\left(\frac{\varphi}{1-\varphi}\right)^{s_{2}}$ & 44 & 12 & $X=\frac{s_{1} s_{2} s_{3} \varphi}{\left(1-s_{3} s_{4} \varphi\right)\left[1+\left(s_{2}-s_{4}\right) s_{3} \varphi\right]}$ & 4 & 32 \\
\hline$X=\frac{s_{1} s_{2 \varphi}}{(1-\varphi)\left[1+\left(s_{2}-1\right) \varphi\right]}$ & 33 & 13 & $X=s_{1} \exp \left(s_{2} h \varphi\right)+s_{3} \varphi^{s_{4}}$ & 46 & 33 \\
\hline $\ln (\varphi)=\frac{-s_{1} s_{2} x}{\left(1 / S_{3}\right)-(1 / T)-1}$ & 2 & 14 & $\varphi=\exp \left[\mathrm{s}_{1} \exp \left(\mathrm{s}_{2}-\mathrm{s}_{3} \mathrm{~T}\right) \mathrm{X}^{\mathrm{s}_{4}}\right.$ & 16 & 34 \\
\hline$X=\frac{\mathrm{s}_{1} \mathrm{~s}_{2 \varphi}\left(-\varphi^{\mathrm{s}_{3}}\right)}{(1-\varphi)\left[1+\left(\mathrm{s}_{2}-1\right) \varphi\right]}$ & 49 & 15 & $\varphi=s_{1}+s_{2} X+s_{3} X^{2}+s_{4} X^{3}+s_{5} X^{4}$ & 45 & 35 \\
\hline $\mathrm{X}=\frac{1}{\mathrm{~s}_{2}} \mathrm{~h}\left[\frac{1}{\mathrm{~s}_{1}}\left(\mathrm{~h} \varphi-\mathrm{s}_{3}\right)\right]$ & 13 & 16 & $h\left[\varphi P_{s}(T)\right]=\left(s_{1}+s_{2} X\right) \ln \left(P_{s}(T)+\left(s_{3}+s_{4} X+s_{5} X^{2}\right)\right.$ & 28 & 36 \\
\hline$\frac{\varphi}{X}=s_{1}+s_{2} \varphi+s_{3} \varphi^{2}$ & 23 & 17 & $h(\varphi)=-\frac{s_{1}}{\mathbb{R}} \exp \left(-s_{2} X\right)$ & 54 & 37 \\
\hline$h\left[X+\left(X^{2}+s_{1}\right)^{0.5}\right]=s_{2} \varphi+s_{3}$ & 31 & 18 & $h\left[\varphi P_{s}(T)\right]=s_{1}+s_{2} X-\left(s_{3}+s_{4} X+\frac{s_{5}}{X}+\frac{s_{6}}{X^{2}}\right) / T$ & 62 & 38 \\
\hline$X=s_{1}\left(\frac{\varphi}{s_{3}-\varphi}\right)^{s_{2}}$ & 21 & 19 & $1-\varphi=\exp \left[-s_{1}\left(s_{5} T+s_{6}\right)^{s_{2}}(100 X)^{s_{3}}\left(s_{5} T+s_{6}\right)^{s_{4}}\right]$ & 17 & 39 \\
\hline$X=\frac{s_{1} s_{2} s_{3} \varphi}{\left(1-s_{3} \varphi\right)\left[1+\left(s_{2}-1\right) s_{3} \varphi\right]}$ & 59 & 20 & $\begin{array}{c}X=\left(-s_{4} T+s_{5}\right) \frac{\left[\exp \left(\frac{s_{1}}{T}+s_{2}\right)\right]\left(s_{5} T+s_{6}\right)}{(1-K \varphi)[1+(C-1) K \varphi]} \\
K=\mathbb{S}_{5}+S_{6} \quad C=\exp \left(\frac{s_{1}}{T}+s_{2}\right)\end{array}$ & 43 & 40 \\
\hline
\end{tabular}

Ref corresponds to: 2 - AGUERRE, SUAREZ \& VIOLLAZ (1983); 4 - ANDERSON \& HALL (1948); 7 - BRADLEY (1936); 9 - BRUNAUER, EMMETT \& TELLER (1938); 12 - CAURIE (1970); 13 - CHEN (1971); 14 - CHEN \& CLAYTON (1971); 15 - CHUNG \& PFOST (1967); 16 - COSTA, MURATA \& BARROZO (1997); 17 - DAY \& NELSON (1965); 21 - GINZBURG \& SAVINA (1982); 23 - HAILWOOD \& HORROBIN (1946); 24 - HALSEN (1948); 25 - HARKINS \& JURA (1944a); 26 - HARKINS \& JURA (1944b); 28 - HAYNES (1978); 29 - HENDERSON (1952); 30 - HUTTIG (1948); 31 - IGLESIAS \& CHERIFE (1976b); 33 - JAAFAR \& MICHALOWSKI (1990); 37 - KUHN (1964); 38 - LANGMUIR (1916); 41 - MADAMBA, DRISCOLL \& BUCKLE (1995); 42 - MIZRAHI, LABUZA \& KAREL (1970); 43 - MOTARJEMI (1988); 44 - OSWIN (1946); 45 - PAKOWSKI (1995); 46 - PELEG (1992); 49 - ROUNSLEY (1961); 54 - STROHMAN \& YOERGER (1967); 59 - VAN DEN BERG (1983); 62 - WERLING (1978). 


\section{RESULTS AND DISCUSSION}

Table 2 and 3 present the agreements obtained for several products. The references of the experimental data, the number of experimental points, temperature and range of relative humidity are also presented in such tables. Besides the products in Table 2, it was tried to adjust data from isotherms of fat cattle (TRUJILLO, YEOW \& PHAM 2003) but, no model presented $r^{2} \geq 0.99$ for this product. Trujillo, Yeow \& Pham (2003) presented adjust for fat cattle with GAB (VAN DEN BERG, 1983) equation, but did not present $r^{2}$ values.

TABLE 2 - AGREEMENT PARAMETERS OF MATHEMATICAL MODELS TO EXPERIMENTAL DATA OF SORPTION ISOTHERMS OF SEVERAL FOOD PRODUCTS

\begin{tabular}{|c|c|c|c|c|c|c|c|c|c|c|}
\hline Product (Ref.), NP & $\begin{array}{l}\mathrm{T} \\
{[\mathrm{K}]}\end{array}$ & $\varphi$ & Eq. & $\mathrm{S}_{1}$ & $\mathrm{~S}_{2}$ & $\mathrm{~S}_{3}$ & $\mathrm{~S}_{4}$ & $\mathrm{~S}_{5}$ & $\begin{array}{l}r^{2} \\
\times 10^{4} \\
\end{array}$ & $\begin{array}{l}\mathrm{S} E \\
\times 10^{2} \\
\end{array}$ \\
\hline \multirow{3}{*}{$\begin{array}{l}\text { Açai }{ }^{\mathrm{a}(52), 12 \text { for each }} \\
\text { temperature }\end{array}$} & 288 & $0.13-0.96$ & 17 & 0.331345 & -1.63259 & 5.323191 & - & - & 9558 & 5.56 \\
\hline & 298 & $0.11-0.93$ & 17 & 0.259707 & -1.05888 & 4.403736 & - & - & 9365 & 6.45 \\
\hline & 308 & $0.10-0.93$ & 17 & 0.280826 & -1.53972 & 6.031590 & - & - & 9761 & 4.58 \\
\hline \multirow{3}{*}{$\begin{array}{l}\text { Açaid(52),12 for each } \\
\text { temperature }\end{array}$} & 288 & $0.14-0.96$ & 17 & 0.645017 & -4.40031 & 10.97147 & - & - & 9887 & 2.53 \\
\hline & 298 & $0.12-0.93$ & 17 & 0.542244 & -3.41149 & 9.210610 & - & - & 9735 & 4.60 \\
\hline & 308 & $0.14-0.93$ & 17 & 0.438836 & -2.86611 & 8.810009 & - & - & 9772 & 4.04 \\
\hline Alginic acid(51),5 & 298 & $0.34-1.00$ & 13 & 0.0587 & $1.9265 \times 10^{-6}$ & 0.8048 & - & - & 9965 & 0.46 \\
\hline $\begin{array}{l}\text { Amaranth } \\
\text { seeds } \text { s }^{(47), 7}\end{array}$ & 308 & $0.03-0.98$ & 15 & 0.3974 & 1.3899 & 0.3421 & - & - & 9963 & 0 \\
\hline Apple ${ }^{a(34), 10}$ & 303 & $0.11-0.90$ & 13 & 0.1736 & 8.645 & 0.9083 & - & - & 9947 & 1.81 \\
\hline Apple $^{\mathrm{d}(34), 10}$ & 303 & $0.11-0.90$ & 35 & 0.6469 & -6.7077 & 26.8163 & -33.8385 & 13.9568 & 9924 & 2.19 \\
\hline \multirow{2}{*}{$\begin{array}{l}\text { Apricot } \\
\text { each temperature }\end{array}$} & 303 & $0.11-0.90$ & 13 & 0.0920 & 6.2722 & 0.9907 & - & - & 9989 & 0.76 \\
\hline & 318 & $0.11-0.88$ & 4 & -0.096 & -0.0155 & - & - & - & 9964 & 1.29 \\
\hline \multirow{2}{*}{ Apricot ${ }^{(34), 30}$} & 303 & $0.11-0.90$ & 4 & -0.1026 & 0.0896 & - & - & - & 9957 & 1.81 \\
\hline & 318 & $0.11-0.88$ & 13 & 0.0778 & 10.7716 & 1.0314 & - & - & 9979 & 1.07 \\
\hline Banana ${ }^{(35), 29}$ & 318 & $0.03-0.39$ & 35 & 0.4044 & -2.069 & 36.5398 & -130.933 & 147.3786 & 9664 & 2.25 \\
\hline Barley ${ }^{(8), 10}$ & 298 & $0.10-1.00$ & 33 & 0.1827 & 0.5758 & 0.183 & 8.9122 & & 9996 & 0.17 \\
\hline $\begin{array}{l}\text { Black bean } \\
\text { seed, } \\
\text { CAMILO(11), } 5\end{array}$ & 298 & $0.58-0.93$ & 12 & 0.0896 & 0.5981 & - & - & - & 9995 & 0.18 \\
\hline $\begin{array}{l}\text { Black bean } \\
\text { seed, } \\
\text { JEO }^{(11), 5}\end{array}$ & 298 & $0.58-0.93$ & 12 & 0.0860 & 0.626 & - & - & - & 9980 & 0.36 \\
\hline $\begin{array}{l}\text { Black bean } \\
\text { seed, } \\
\text { JEO2(11),5 }\end{array}$ & 298 & $0.58-0.93$ & 12 & 0.0870 & 0.6149 & - & - & - & 9995 & 0.18 \\
\hline $\begin{array}{l}\text { Black bean } \\
\text { seed, } \\
\text { NAG12(39),5 }\end{array}$ & 298 & $0.58-0.93$ & 12 & 0.0887 & 0.6119 & - & - & - & 9963 & 0.68 \\
\hline $\begin{array}{l}\text { Black bean } \\
\text { seed, } \\
\text { TUC500(11),5 }\end{array}$ & 298 & $0.58-0.93$ & 12 & 0.0897 & 0.5997 & - & - & - & 9988 & 0.37 \\
\hline Buckwheat $^{(8), 10}$ & 298 & $0.10-1.00$ & 13 & 0.0829 & 19.2425 & 0.7431 & - & - & 9940 & 0.59 \\
\hline $\begin{array}{c}\text { Coffee } \\
\text { cherry }^{\mathrm{d}(1), 4}\end{array}$ & 298 & $0.20-0.75$ & 17 & -5.391 & 45.7164 & -49.4577 & - & - & 9931 & 2.10 \\
\hline Colza $(60)$ & 298 & $0.10-0.90$ & 17 & 198.74 & 18.8988 & -079 & - & - & 9951 & 0.19 \\
\hline $\begin{array}{c}\text { Coffee } \\
\text { products } \\
\text { FDDC } C^{(27), 8}\end{array}$ & 293 & $0.00-0.60$ & 35 & -0.015 & 1.3095 & 66.4305 & -323.701 & 30.4328 & 9770 & 3.11 \\
\hline $\begin{array}{l}\text { Coffee } \\
\text { products } \\
\text { FDUC }\end{array}$ & 293 & $0.00-0.61$ & 13 & 0.0396 & 22.8429 & 1.2584 & - & - & 9976 & 0.23 \\
\hline $\begin{array}{l}\text { Coffee } \\
\text { products } \\
\text { RGDC }^{(27), 8}\end{array}$ & 293 & $0.00-0.61$ & 35 & 0.0740 & -13.801 & 939.2347 & -10572.7 & $2.20 \times 10^{4}$ & 9638 & 12.31 \\
\hline $\begin{array}{c}\text { Coffee } \\
\text { products } \\
\text { RGUC } \\
\text { RG),8 }\end{array}$ & 293 & $0.00-0.61$ & 35 & -041 & 24.8236 & $-1.3 \times 10^{3}$ & $2.94 \times 10^{4}$ & $-1.9 \times 10^{5}$ & 9666 & 3.74 \\
\hline
\end{tabular}




\begin{tabular}{|c|c|c|c|c|c|c|c|c|c|c|}
\hline Product (Ref.), NP & $\begin{array}{l}\mathrm{T} \\
{[\mathrm{K}]}\end{array}$ & $\varphi$ & Eq. & $\mathrm{S}_{1}$ & $\mathrm{~S}_{2}$ & $\mathrm{~S}_{3}$ & $\mathrm{~S}_{4}$ & $\mathrm{~S}_{5}$ & $\begin{array}{l}r^{2} \\
\times 10^{4} \\
\end{array}$ & 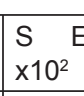 \\
\hline $\begin{array}{c}\text { Coffee } \\
\text { products } \\
\text { SDDC(27),8 }\end{array}$ & 293 & $0.00-0.61$ & 13 & 0.0332 & 23.6737 & 1.3089 & - & - & 9933 & 0.37 \\
\hline $\begin{array}{l}\text { Coffee } \\
\text { products } \\
\text { SDUC(27),8 }\end{array}$ & 293 & $0.00-0.61$ & 13 & 0.0341 & 25.5487 & 1.3159 & - & - & 9903 & 0.46 \\
\hline Cotton seed ${ }^{(8), 6}$ & 298 & $0.10-1.00$ & 4 & -0.020 & 0.0568 & - & - & - & 9977 & 0.28 \\
\hline \multirow{3}{*}{$\begin{array}{l}\text { Cowpea(3), } 8 \text { for } \\
\text { each temperature }\end{array}$} & 313 & $0.43-0.98$ & 35 & 0.6089 & -6.8852 & 65.1851 & 12.5994 & -599.481 & 9984 & 0.77 \\
\hline & 333 & $0.43-0.95$ & 35 & 1.0707 & -24.8579 & 303.7234 & -1237.9 & 1629.44 & 9987 & 0.69 \\
\hline & 353 & 0.37-0.99 & 35 & 1.0707 & -24.8579 & 303.7234 & -1237.9 & 1629.44 & 9987 & 0.69 \\
\hline \multirow{3}{*}{$\begin{array}{l}\text { Cupuaçua(52),12 } \\
\text { for each temperature }\end{array}$} & 288 & $0.18-0.94$ & 17 & 0.121477 & 0.584706 & 0.465717 & - & - & 9323 & 5.50 \\
\hline & 398 & $0.13-0.94$ & 17 & 0.178998 & 0.071454 & 1.405506 & - & - & 9533 & 6.03 \\
\hline & 308 & $0.10-0.95$ & 17 & 0.199202 & -0.028952 & 1.460395 & - & - & 9562 & 5.86 \\
\hline \multirow{3}{*}{$\begin{array}{l}\text { Cupuaçud(52),12 } \\
\text { for each temperature }\end{array}$} & 288 & $0.27-0.94$ & 17 & 0.188790 & 0.267102 & 0.809608 & - & - & 9834 & 3.06 \\
\hline & 398 & $0.08-0.94$ & 17 & 0.253008 & -0.391976 & 2.026859 & - & - & 9462 & 5.78 \\
\hline & 308 & $0.11-0.95$ & 17 & 0.246861 & -0.218345 & 1.625365 & - & - & 9511 & 6.50 \\
\hline $\begin{array}{c}\text { Dry } \\
\text { beans, dark } \\
\text { red kidney }{ }^{(8), 8}\end{array}$ & 298 & $0.10-1.00$ & 13 & 0.0735 & 25.8184 & 0.8516 & - & - & 9972 & 0.28 \\
\hline $\begin{array}{c}\text { Dry beans, } \\
\text { flat,small } \\
\text { white }^{(8), 8}\end{array}$ & 298 & $0.10-1.00$ & 32 & 0.0737 & 5.7404 & 5.6272 & 0.1491 & - & 9994 & 0.12 \\
\hline $\begin{array}{l}\text { Dry beans, } \\
\text { great } \\
\text { Northern }\end{array}$ & 298 & $0.10-1.00$ & 32 & 0.0741 & 5.9422 & 5.6744 & 0.147 & - & 9998 & 0.07 \\
\hline $\begin{array}{l}\text { Dry beans, } \\
\text { light red } \\
\text { kidney }^{(8), 8}\end{array}$ & 298 & $0.10-1.00$ & 13 & 0.0755 & 35.4002 & 0.8377 & - & - & 9997 & 0.09 \\
\hline $\begin{array}{l}\text { Dry beans, } \\
\text { pinto }^{(8), 8}\end{array}$ & 298 & $0.10-1.00$ & 13 & 0.0748 & 35.5077 & 0.8343 & - & - & 9993 & 0.13 \\
\hline $\begin{array}{l}\text { Dry beans, red } \\
\text { Mexican }^{(8), 8}\end{array}$ & 298 & $0.10-1.00$ & 13 & 0.0743 & 36.3581 & 0.8479 & - & - & 9997 & 0.09 \\
\hline Soy bran ${ }^{(40), 6}$ & 323 & $0.11-0.80$ & 13 & 0.042 & 20.8406 & 1.0362 & - & - & 9988 & 0.26 \\
\hline Flaxseed $^{(8), 10}$ & 298 & $0.10-1.00$ & 13 & 0.0439 & 37.1743 & 0.8392 & - & - & 9984 & 0.27 \\
\hline Flour $^{(10), 6}$ & 293 & $0.12-0.89$ & 13 & 0.0681 & 117.2592 & 0.8163 & - & - & 9978 & 0.29 \\
\hline Gluten $^{(10), 6}$ & 293 & $0.07-0.92$ & 35 & -0.277 & 9.839 & -24.6865 & 18.6295 & -2.5862 & 9924 & 2.72 \\
\hline Tragacanth(10),4 & 298 & $0.34-1.00$ & 9 & 0.035 & -2.5624 & - & - & - & 9979 & 0.41 \\
\hline Grape $^{a(34), 10}$ & 303 & $0.11-0.90$ & 21 & 1.0036 & 0.1049 & 1.3012 & - & - & 9975 & 1.25 \\
\hline Grape $^{d(34), 10}$ & 303 & $0.11-0.90$ & 21 & 1.0064 & 0.1282 & 1.3505 & - & - & 9985 & 0.99 \\
\hline Kiwi(35),28 & 298 & $0.27-0.88$ & 35 & 0.3662 & -2.6812 & 17.237 & -28.044 & 14.1876 & 9673 & 3.68 \\
\hline Lean beef(58), 10 & 278 & $0.34-0.98$ & 12 & 0.1069 & 0.6025 & - & - & - & 9995 & 0.85 \\
\hline $\begin{array}{l}\text { Mushroom, } \\
\text { Agaricus }^{(50), 5}\end{array}$ & 303 & $0.22-0.40$ & 13 & 083 & 3.9918 & 0.7453 & - & - & 9992 & 0.26 \\
\hline $\begin{array}{c}\text { Mushroom, } \\
\text { Pleurotus }^{(50), 5}\end{array}$ & 303 & $0.22-0.40$ & 13 & 0.1074 & 3.3038 & 0.776 & - & - & 9942 & 0.52 \\
\hline Oats ${ }^{(8), 10}$ & 298 & $0.10-1.00$ & 13 & 0.0724 & 17.1016 & 0.7719 & - & - & 9944 & 0.58 \\
\hline $\begin{array}{l}\text { Peanuts, } \\
\text { kernels }^{(8), 6}\end{array}$ & 283 & $0.10-1.00$ & 5 & 0.1484 & 0.0034 & & - & - & 9900 & 1.70 \\
\hline $\begin{array}{c}\text { Peanuts, } \\
\operatorname{pod}^{(8), 6}\end{array}$ & 283 & $0.10-1.00$ & 13 & 0.0502 & 15.6282 & 0.7986 & - & - & 9968 & 0 \\
\hline Pear ${ }^{(35), 30}$ & 298 & $0.21-0.91$ & 35 & 0.1976 & 0.5592 & 7.8229 & -19.886 & 12.8643 & 9608 & 4.43 \\
\hline \multirow{3}{*}{$\begin{array}{c}\text { Pink } \\
\text { Shrimp } \\
\text { a(5),24 }\end{array}$} & 283 & $0.07-0.92$ & 17 & 0.332459 & -1.32220 & 4.247144 & - & - & 9495 & 5.28 \\
\hline & 298 & $0.24-0.94$ & 17 & 0.244509 & -0.383894 & 2.091547 & - & - & 9760 & 3.07 \\
\hline & 313 & $0.13-0.91$ & 17 & 0.299176 & -1.02548 & 3.786085 & - & - & 9590 & 4.29 \\
\hline \multirow{3}{*}{$\begin{array}{c}\text { Pink } \\
\text { Shrimp } \\
\text { d(5),24 }^{(24}\end{array}$} & 283 & 0.06-0.92 & 17 & 0.484350 & -2.23579 & 5.696980 & - & - & 9788 & 3.79 \\
\hline & 298 & 0.09-0.94 & 17 & 0.380162 & \begin{tabular}{|l|}
-1.18178 \\
\end{tabular} & 3.348690 & - & - & 9721 & 4.33 \\
\hline & 313 & $0.15-0.91$ & 17 & 0.439139 & -1.97821 & 5.465058 & - & - & 9856 & 3.12 \\
\hline
\end{tabular}




\begin{tabular}{|c|c|c|c|c|c|c|c|c|c|c|}
\hline Product (Ref.), NP & $\begin{array}{l}\mathrm{T} \\
{[\mathrm{K}]}\end{array}$ & $\varphi$ & Eq. & $\mathrm{S}_{1}$ & $\mathrm{~S}_{2}$ & $\mathrm{~S}_{3}$ & $\mathrm{~S}_{4}$ & $\mathrm{~S}_{5}$ & $\begin{array}{l}r^{2} \\
\times 10^{4}\end{array}$ & $\begin{array}{l}S E \\
x 10^{2}\end{array}$ \\
\hline \multirow{5}{*}{$\begin{array}{l}\text { Potato }{ }^{\mathrm{a}(34),}, 10 \text { for } \\
\text { each temperature }\end{array}$} & 303 & $0.11-0.90$ & 21 & 1.0771 & 0.0573 & 1.165 & - & - & 9977 & 1.22 \\
\hline & 318 & $0.11-0.88$ & 35 & -0.303 & 13.038 & -58.0187 & 117.795 & -80.5684 & 9977 & 1.18 \\
\hline & 333 & $0.11-0.84$ & 15 & 278.48 & 0.3217 & 0.0009 & - & - & 9907 & 0.49 \\
\hline & 303 & $0.11-0.90$ & 21 & 0.9919 & 0.0309 & 1.5213 & - & - & 9964 & 1.51 \\
\hline & 318 & $0.11-0.88$ & 13 & 0.0604 & 21.4821 & 0.9305 & - & - & 9950 & 0.61 \\
\hline $\begin{array}{c}\text { Potato } \\
\text { starch }^{(51), 5}\end{array}$ & 298 & $0.34-1.00$ & 13 & 0.1003 & 1811190.1 & 0.7174 & - & - & 9994 & 0.20 \\
\hline $\begin{array}{c}\text { Quinoa } \\
\text { grains }^{(57), 10}\end{array}$ & 293 & $0.09-0.85$ & 35 & 1.2616 & -54.2659 & 806.5031 & -4368 & 8207.42 & 9981 & 1.08 \\
\hline Rice, $\operatorname{milled}^{(8), 10}$ & 298 & $0.10-1.00$ & 35 & 0.6081 & -22.1115 & 298.4938 & -1248.2 & 1705.60 & 9937 & 2.28 \\
\hline Rice, rough ${ }^{(8), 8}$ & 273 & $0.10-1.00$ & 13 & 0.0979 & 19.7848 & 0.6592 & & & 9953 & 0.31 \\
\hline $\begin{array}{l}\text { Rice, whole } \\
\text { grain(8),10 }\end{array}$ & 298 & $0.10-1.00$ & 35 & -0.516 & 18.8067 & -220.677 & 1410.02 & -2986.71 & 9995 & 0.60 \\
\hline Rye $^{(8), 9}$ & 298 & $0.10-1.00$ & 12 & 0.1218 & 0.3331 & - & - & - & 9940 & 0.45 \\
\hline $\begin{array}{l}\text { Shelled corn } \\
\text { WD }^{(8), 10}\end{array}$ & 298 & $0.10-1.00$ & 21 & 1.2817 & 0.0431 & 1.472 & - & - & 9924 & 2.50 \\
\hline $\begin{array}{l}\text { Shelled corn } \\
\text { YD }^{(8), 5}\end{array}$ & 266 & $0.10-1.00$ & 13 & 0.1211 & 5.3526 & 0.5758 & - & - & 9996 & 0.06 \\
\hline $\begin{array}{c}\text { Shelled } \\
\text { popcorn }^{(8), 10}\end{array}$ & 298 & $0.10-1.00$ & 35 & 0.5162 & -20.1887 & 293.0418 & -1274.7 & 1800.92 & 9981 & 1.24 \\
\hline Sorghum(8),7 & 272 & $0.10-1.00$ & 21 & 8.5874 & 0.8074 & 0.6427 & - & - & 9976 & 0.83 \\
\hline $\begin{array}{l}\text { Sorghum, } \\
\text { kafir( }{ }^{(8), 8}\end{array}$ & 277 & $0.10-1.00$ & 21 & 1.4962 & 0.0668 & 1.4249 & - & - & 9996 & 0.47 \\
\hline Soybeans ${ }^{(8), 8}$ & 298 & $0.10-1.00$ & 13 & 0.052 & 50.5255 & 0.8654 & - & - & 9971 & 0.30 \\
\hline $\operatorname{Starch}^{(10), 6}$ & 293 & $0.10-0.92$ & 21 & 1.4754 & 0.1099 & 1.1014 & - & - & 9972 & 1.46 \\
\hline \multirow{2}{*}{$\begin{array}{l}\text { Sugar beet } \\
\text { seeds }^{(8), 8}\end{array}$} & 277 & $0.30-1.00$ & 12 & 0.1425 & 0.3174 & - & - & - & 9933 & 0.46 \\
\hline & 288 & $0.30-1.00$ & 12 & 0.1273 & 0.3027 & - & - & - & 9993 & 0.13 \\
\hline $\begin{array}{c}\text { West Indian } \\
\text { cherry, Pulp } \\
(53), 7\end{array}$ & 303 & $0.19-0.95$ & 13 & 0.1494 & 92.0271 & 1.0682 & - & - & 9954 & 1.83 \\
\hline Wheat $^{(8), 7}$ & 272 & $0.10-1.00$ & 13 & 0.1238 & 5.4292 & 0.5437 & - & - & 9980 & 0.17 \\
\hline $\begin{array}{c}\text { Wheat, } \\
\text { durum }{ }^{(8), 10}\end{array}$ & 298 & $0.10-1.00$ & 13 & 0.0665 & 60.6882 & 0.8151 & - & - & 9936 & 0.69 \\
\hline $\begin{array}{l}\text { Wheat, hard } \\
\text { red spring } \\
(8), 10\end{array}$ & 298 & $0.10-1.00$ & 13 & 0.0802 & 15.4221 & 0.7601 & - & - & 9955 & 0.54 \\
\hline $\begin{array}{l}\text { Wheat, hard } \\
\text { red winter }\end{array}$ & 298 & $0.10-1.00$ & 13 & 0.0793 & 15.7338 & 0.763 & - & - & 9960 & 0.52 \\
\hline $\begin{array}{l}\text { Wheat, soft } \\
\text { red winter }{ }^{(8), 5}\end{array}$ & 266 & $0.10-1.00$ & 13 & 0.1324 & 6.3960 & 0.5226 & - & - & 9981 & 0.12 \\
\hline $\begin{array}{l}\text { Wheat, } \\
\text { white }^{(8), 10}\end{array}$ & 298 & $0.10-1.00$ & 13 & 0.0710 & 37.2703 & 0.8000 & - & - & 997 & 0.47 \\
\hline
\end{tabular}

Ref corresponds to 1 - AFONSO Jr (2001), 3 - AJIBOLA, AVIARA \& AJETUMOBI (2003), 5 - ASSUNÇÃO \& PENA (2007), 8 - BROOKER et al. (1974), 10 - BUSHUK \& WINKLER (1957), 11 - CASTILLO et al. (2003), 27 - HAYAKAWA, MATAS \& HWANG (1978), 34 - KAYMAK-ERTEKIN \& GEDIK (2004), 35 - KIRANAUDIS et al. (1997), 40 - LUZ et al. (2006), 47 - POLLIO, TOLABA \& SUAREZ (1988), 50 - SHIVHAREA et al. (2004), 51 - SHOTTON \& HARB (1965), 52 - SILVA, SILVA \& PENA (2008), 53 - SILVA et al. (2005), 57 - TOLABA et al. (2004), 58 - TRUJILLO, YEOW \& PHAM (2003), 60 - VASCONCELOS (1998), NP: number of experimental points; a: Adsorption; d: Desorption; FDUC: freeze dried undecaffeinated; FDDC: freeze dried decaffeinated; SDUC: spray dried undecaffeinated; SDDC: spray dried decaffeinated; RGUC: roast \& ground undecaffeinated; RGDC: roast \& ground decaffeinated.

Table 2 shows that, among the models that do not consider temperature or vapor pressure as variables, the one that presented the higher number of agreement was Jaafar \& Michalowski (1990) model, equation 13, followed by equations $35,12,32,17,4,15,33,9$ and 5 , in this order. The great fitness of Jaafar and Michalowski (1990) model should be because it is a phenomenological modification of BET model (BRUNAUER, EMMETT \& TELLER, 1938). That model was also among the best ones in a study with dry residue of pink shrimp, developed by Assunção and Pena (2007). Even thought equation 35 presented very good agreements; it is a polynomial equation without phenomenological basis. Due to this fact, it was only presented in Table 2 for cases where no other model carried out to $r^{2} \geq 0.99$. 
TABLE 3 - AGREEMENT PARAMETERS OF MATHEMATICAL MODELS OF ISOTHERMS WITH TEMPERATURE ANDIOR VAPOR PRESSURE CONSIDERED AS VARIABLES

\begin{tabular}{|c|c|c|c|c|c|c|c|c|c|}
\hline Product(Ref), NP, RT & Eq. & $\mathrm{S}_{1}$ & $\mathrm{~S}_{2}$ & $\mathrm{~S}_{3}$ & $\mathrm{~S}_{4}$ & $\mathrm{~S}_{5}$ & $\mathrm{~S}_{6}$ & $\begin{array}{l}r^{2} \\
\times 10^{4} \\
\end{array}$ & $\begin{array}{l}\text { SE } \\
\times 10^{2}\end{array}$ \\
\hline Acacia $^{(51), ~ 16, ~ 298-323 ~}$ & 31 & -0.0171 & 6.0912 & -4.4444 & -16.0803 & - & - & 9728 & 2.94 \\
\hline Açai ${ }^{a(52), 36,288-308}$ & 36 & 0.284867 & 0.70117 & -3.27602 & 0.02190 & 2.2033 & - & 9820 & 0.96 \\
\hline $\begin{array}{l}\text { Pulp of West Indian cherry }{ }^{(53),} \\
21,303-323\end{array}$ & 37 & 0.9824 & -3.9542 & -0.0339 & 1.6612 & 0.0249 & - & 9249 & 5.24 \\
\hline Alginic $\operatorname{acid}^{(41), 24,298-323}$ & 31 & & 24.2137 & -5.7191 & -19.147 & - & - & 9772 & 3.51 \\
\hline Amaranth seeds ${ }^{(47), 21,308-338}$ & 31 & 0.9489 & -17.6845 & -6.6602 & -19.855 & - & - & 9890 & 2.43 \\
\hline Apple ${ }^{a(34), 30,303-333}$ & 37 & 7.9293 & -22.4151 & -1.2748 & -2.8965 & 0.0255 & - & 9844 & 0.98 \\
\hline Apple ${ }^{d(34), 30,303-333}$ & 26 & 154.5864 & 4.6377 & -271.3408 & - & - & - & 9617 & 1.53 \\
\hline Apricot ${ }^{\mathrm{a}(34), 30,303-333}$ & 28 & 2.0398 & -0.0002 & 0.0202 & - & - & - & 9803 & 0.97 \\
\hline Apricot $^{\mathrm{d}(34), 30,303-333}$ & 28 & 8.3626 & 0.0005 & 0.2542 & - & - & - & 9655 & 1.47 \\
\hline Banana $^{(35), 56,303-333}$ & 28 & 5.3350 & 0.0037 & 1.2456 & - & - & - & 9662 & 3.05 \\
\hline Beneficed coffee ${ }^{(1), 16,298-328}$ & 28 & $1.316 \times 10^{6}$ & 0.0007 & 0.2801 & - & - & - & 9715 & 0.86 \\
\hline Coffee cherry ${ }^{a(1), 16,298-328}$ & 40 & 30.1010 & -1.989 & - & 0.0005 & 0.0205 & -14.634 & 9752 & 2.28 \\
\hline Coffee cherry ${ }^{d(1), 16,}, 298-328$ & 24 & 0.0014 & 0.937 & 317.6441 & - & - & - & 8838 & 5.84 \\
\hline Cupuaçua(52),36,288-308 & 36 & 2.141804 & -1.80427 & -1.92489 & -3.5011 & 4.7188 & - & 9694 & 2.52 \\
\hline Pelled Coffee ${ }^{a(1), 16,298-328}$ & 37 & 7.2823 & -36.2975 & -0.2594 & 0.1976 & 0.0302 & - & 9426 & 4.97 \\
\hline Pelled Coffee ${ }^{d(1), 16,298-328}$ & 28 & 0.0016 & 0.8589 & 288.6395 & - & - & - & 8913 & 5.31 \\
\hline Pulped Coffee ${ }^{a(1), 16,298-328}$ & 40 & 15.4335 & -1.2370 & - & 0.0003 & 0.0143 & -8.4196 & 9648 & 1.85 \\
\hline Colza ${ }^{(60), 15,298-308}$ & 37 & -0.9789 & -9.1054 & 19.0038 & -5.0495 & $2.5147 \times 10^{7}$ & - & 9789 & 3.16 \\
\hline $\begin{array}{l}\text { Coffee products FDDC } \\
\text { 293-303, }\end{array}$ & 36 & 2.0103 & -7.1813 & -4.6032 & 53.6349 & -174.8835 & - & 9667 & 3.73 \\
\hline $\begin{array}{l}\text { Coffee products } \text { FDUC }^{(27),} \text { 16, } \\
\text { 293-303 }\end{array}$ & 37 & 2.417 & -33.1918 & -0.3692 & 1.8171 & 0.0394 & - & 9793 & 2.95 \\
\hline $\begin{array}{l}\text { Coffee products SDUC } \\
\text { 293-303), }\end{array}$ & 37 & 4.0303 & -47.3896 & -0.7541 & -2.5856 & 0.0863 & - & 9822 & 2.73 \\
\hline Cowpea ${ }^{(3), 40,313-353}$ & 36 & 1.1034 & -0.3719 & -1.9959 & 15.4359 & -29.8465 & - & 9732 & 3.32 \\
\hline Dry beans Michelite ${ }^{(8), 20,277-327}$ & 23 & 0.4725 & 2.4337 & 0.8250 & - & - & - & 9908 & 1.92 \\
\hline Soy $\operatorname{bran}^{(40),}, 18,323-343$ & 31 & -0.2697 & -4.9928 & -4.0915 & -34.6631 & - & - & 9530 & 5.58 \\
\hline Flour ${ }^{(10), 24,293-323}$ & 26 & 515.7182 & 18.7084 & $-2.18 \times 10^{2}$ & - & - & - & 9897 & 2.75 \\
\hline Gluten $^{(10), 24,293-323}$ & 37 & 0.6921 & -22.0328 & -0.0475 & 3.7792 & 0.0001 & - & 9564 & 6.37 \\
\hline Tragacanth ${ }^{(10), 20,298-323}$ & 39 & 3.7426 & -10.2467 & 0.3976 & 2.3615 & 0.0084 & -0.903 & 9857 & 2.45 \\
\hline Grape $^{\mathrm{a}(34), 30,303-333}$ & 37 & 2.9192 & -16.6127 & -0.7433 & -1.9644 & 0.0188 & - & 9858 & 0.93 \\
\hline Grape ${ }^{d(34), 30,303-333}$ & 28 & 6.6902 & -0.0019 & -0.4392 & - & - & - & 9808 & 1.52 \\
\hline $\operatorname{Kiwi}^{(35), 57,303-333}$ & 24 & 0.0998 & -277.275 & 0.9813 & - & - & - & 9254 & 5.19 \\
\hline Lean beef ${ }^{(58), 40,278-313}$ & 37 & 0.9612 & -22.99 & -0.5431 & -3.8554 & 0.0249 & - & 9771 & 3.08 \\
\hline Maize starch ${ }^{(51), 24,298-323}$ & 23 & 0.2064 & 2.1971 & 1.0149 & - & - & - & 9779 & 3.45 \\
\hline Mushroom, agaricus ${ }^{(50), 25,303-343}$ & 31 & 0.5170 & -4.927 & -4.1926 & -7.3901 & - & - & 9942 & 1.65 \\
\hline Mushroom, pleurotus ${ }^{(50), 25,303-343}$ & 39 & 0.0045 & 0.5509 & 0.0031 & 1.6132 & 0.1575 & -8.3019 & 9800 & 3.07 \\
\hline Pear ${ }^{(35), 56,303-333}$ & 37 & 0.2460 & -8.1071 & -0.3757 & -1.0104 & 0.0002 & - & 9229 & 6.47 \\
\hline Pork muscle, ${ }^{(58), 40,298-303}$ & 36 & 0.9631 & -0.0360 & -2.1099 & -3.345 & 5.4891 & - & 9545 & 6.12 \\
\hline Pink shrimp ${ }^{(5), 80,283-313}$ & 36 & 1.085395 & -0.09714 & -2.424 & -1.20040 & 2.9577 & - & 9741 & 1.07 \\
\hline Pink shrimp ${ }^{(5), 80,283-313}$ & 36 & 0.875676 & 0.13382 & -2.6494 & 0.21656 & 1.6116 & - & 9722 & 1.48 \\
\hline Potato ${ }^{a(34), 30,303-333}$ & 39 & 0.0406 & -0.1439 & 0.0057 & 1.4222 & 0.1749 & -8.2301 & 9670 & 1.42 \\
\hline Potato starch ${ }^{(51), 24,298-323}$ & 29 & $7.615 \times 10^{6}$ & -2.3783 & 482.5275 & -0.5949 & - & - & 9900 & 2.32 \\
\hline Quinoa grains ${ }^{(57), 27,293-313}$ & 37 & 0.6834 & -16.0253 & -0.0002 & 8.8652 & 0.0009 & - & 9488 & 5.83 \\
\hline Rice rough(8), 34,273-317 & 31 & 1.2719 & -16.9299 & -8.4443 & -18.7186 & - & - & 9913 & 2.23 \\
\hline
\end{tabular}




\begin{tabular}{|c|c|c|c|c|c|c|c|c|c|}
\hline Product $^{(\text {Ref), NP, RT }}$ & Eq. & $\mathrm{S}_{1}$ & $\mathrm{~S}_{2}$ & $\mathrm{~S}_{3}$ & $\mathrm{~S}_{4}$ & $\mathrm{~S}_{5}$ & $\mathrm{~S}_{6}$ & $\begin{array}{l}\mathrm{r}^{2} \\
\mathrm{x} 10^{4}\end{array}$ & $\begin{array}{l}\text { SE } \\
\times 10^{2}\end{array}$ \\
\hline Rice whole grain $(8), 19,298-311$ & 36 & 1.5354 & -2.3821 & -4.7359 & 38.7978 & -80.5366 & - & 998 & 1.14 \\
\hline Shelled corn YD(8), 156, 273-317 & 29 & 35.1057 & -0.3365 & 0.0046 & 1.4388 & - & - & 9545 & 5.18 \\
\hline Sorghum ${ }^{(8), 48, ~ 272-322 ~}$ & 24 & 0.8339 & -173.105 & 2.4289 & - & - & - & 9886 & 2.43 \\
\hline Sorghum, kafir ${ }^{(38),}, 26,277-305$ & 37 & 0.6258 & -10.7376 & -8.2614 & -15.4848 & 5.8267 & - & 9942 & 1.90 \\
\hline $\operatorname{Starch}^{(10), 24,293-323}$ & 29 & 14.8592 & -0.1934 & 0.0058 & 1.3874 & - & - & 9906 & 2.66 \\
\hline Sugar beet seeds ${ }^{(8), 28,277-310}$ & 31 & 1.1242 & -12.0045 & -7.1809 & -16.6978 & - & - & 9953 & 1.38 \\
\hline Wheat ${ }^{(8),}, 110,272-356$ & 31 & 0.7917 & -13.7295 & -6.1830 & -16.265 & - & - & 9704 & 3.88 \\
\hline $\begin{array}{l}\text { Wheat (soft red winter) }{ }^{(8), 29,266-} \\
298\end{array}$ & 26 & 594.0084 & 18.0655 & -203.2337 & & - & - & 9948 & 1.47 \\
\hline Wheat starch ${ }^{(47), 24,298-323}$ & 30 & $2.3692 \times 10^{7}$ & -2.1971 & 253.8559 & -0.8206 & - & - & 9891 & 2.42 \\
\hline
\end{tabular}

Ref corresponds to 1 - AFONSO Jr (2001), 3 - AJIBOLA, AVIARA \& AJETUMOBI (2003), 5 - ASSUNÇÃO \& PENA (2007), 8 - BROOKER et al. (1974), 10 - BUSHUK \& WINKLER (1957), 11 - CASTILLO et al. (2003), 27 - HAYAKAWA, MATAS \& HWANG (1978), 34 - KAYMAK-ERTEKIN and GEDIK (2004), 35 - KIRANAUDIS et al. (1997), 40 - LUZ et al. (2006), 47 - POLLIO, TOLABA \& SUAREZ (1988), 50 - SHIVHAREA et al. (2004), 51 - SHOTTON \& HARB (1965), 52 - SILVA, SILVA \& PENA (2008), 53 - SILVA et al. (2005), 57 - TOLABA et al. (2004), 58 - TRUJILLO, YEOW \& PHAM (2003),

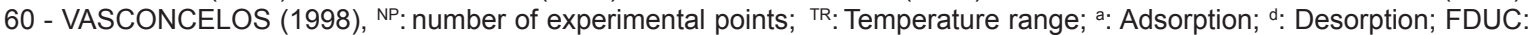
freeze dried undecaffeinated; FDDC: freeze dried decaffeinated; SDUC: spray dried undecaffeinated; SDDC: spray dried decaffeinated; RGUC: roast \& ground undecaffeinated; RGDC: roast \& ground decaffeinated.

Table 3 shows the best agreements obtained with the equations that consider T and/or Ps as variables. According to Table 3, among the models that take in account temperature or vapor pressure as a variable, the one with the best agreements for a product majority was the Strohman and Yoerger (1967) model, equation 31. This model was the best one of rice, as reported by SUN (1999) and one of the best models (SUN and WOODS, 1993) in studies of isotherms of wheat.

The agreement of ROSS equation, as proposed by Roman et al. (2004) was tested here for some food sorption isotherms (Table 4). Even though this equation was based on thermodynamics aspects, considering the components of food products, the agreement was not so good.

Figure 1 shows adjustments of isotherms of corn grains (yd) with Jaafar \& Michalowski (1990) equation and Figure 2 with Chen and Clayton (1971) equation (equations 13 and 29, Table 1 , respectively) in several temperatures. These equations were the best for this product. It is possible to see in these Figures and in Tables 2 and 3, a very good agreement.

TABLE 4 - AGREEMENT PARAMETERS OF ROSS EQUATION

\begin{tabular}{|c|c|c|c|}
\hline Product(Ref.) & $\mathrm{T}[\mathrm{K}]$ & $R^{2} \times 10^{4}$ & SE $\times 10^{2}$ \\
\hline Apricot $^{(34)}$ & 303.15 & 3117 & 60.76 \\
\hline \multirow[t]{5}{*}{ Cowpea ${ }^{(3)}$} & 313.15 & 4006 & 86.75 \\
\hline & 323.15 & 4171 & 90.34 \\
\hline & 333.15 & 5669 & 93.65 \\
\hline & 343.15 & 4370 & 96.05 \\
\hline & 353.15 & 4674 & 97.75 \\
\hline \multirow[t]{3}{*}{ Potato(34) } & 333.15 & 5627 & 72.47 \\
\hline & 318.15 & 5938 & 74.00 \\
\hline & 303.15 & 6342 & 83.97 \\
\hline Rice, milled(8) & 298.15 & 9999 & 73.53 \\
\hline
\end{tabular}

Ref. corresponds to 3 - AJIBOLA, AVIARA \& AJETUMOBI (2003), 8 - BROOKER et al. (1974), 34 - KAYMAK-ERTEKIN and GEDIK (2004). 


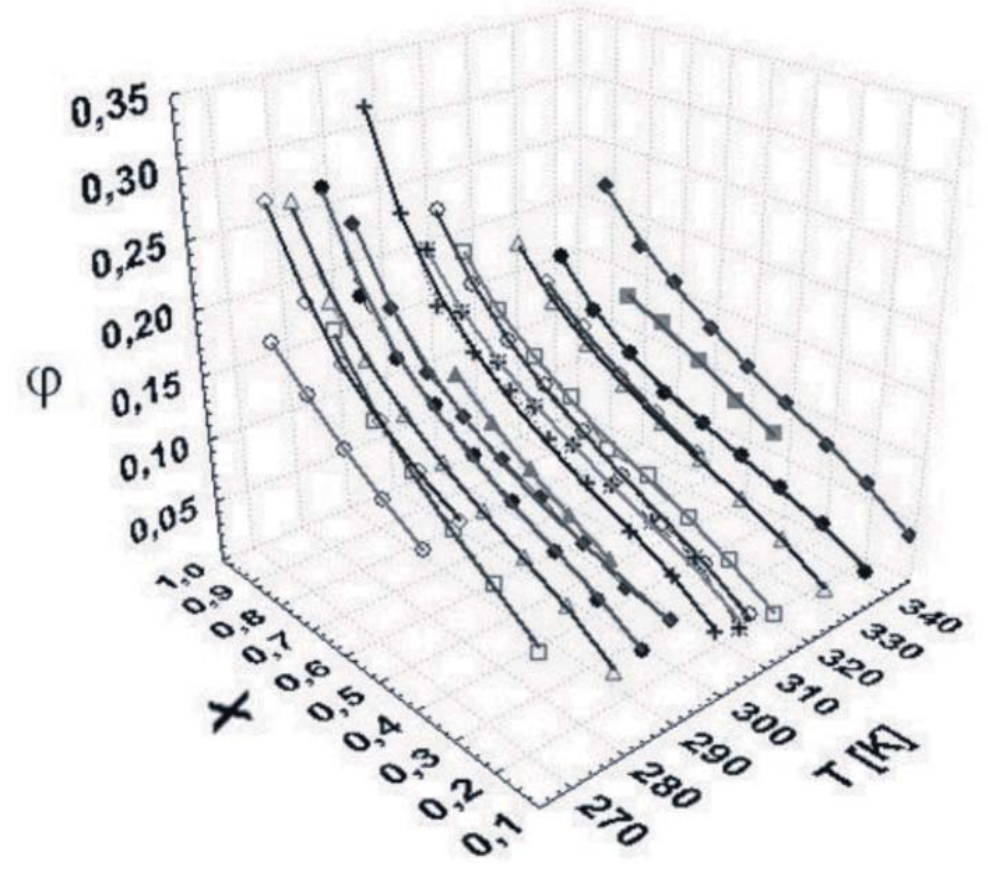

FIGURE 1 - ADJUSTMENTS OF SORPTION ISOTHERMS OF CORN GRAIN YD AT SEVERAL TEMPERATURES WITH JAAFAR \& MICHALOWSKI (1990) EQUATION

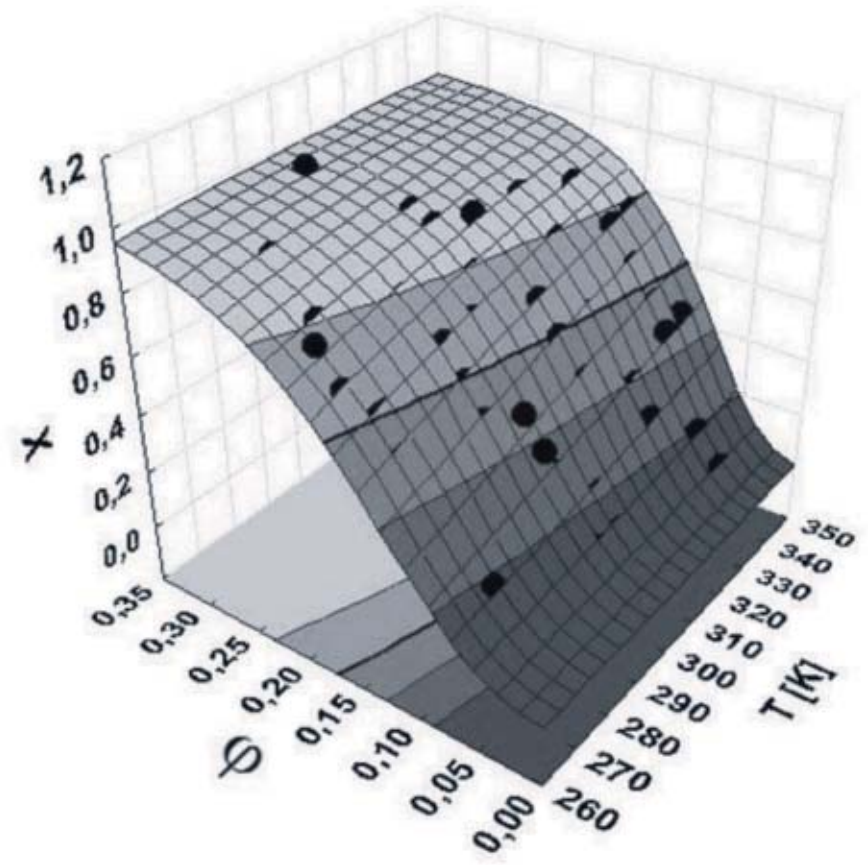

FIGURE 2 - ADJUSTMENTS OF SORPTION ISOTHERMS OF CORN GRAIN YD AT SEVERAL TEMPERATURES WITH CHEN \& CLAYTON (1971) EQUATION 


\section{CONCLUSION}

Among the mathematical models tested for food products, the one of Jaafar and Michalowski was the one with the greater number of best agreements, with respect to models that do not consider temperature or saturation pressure as a variable. It is interesting to note that this model is a phenomenological modification of BET model. Among the models that consider temperature or saturation pressure as a variable, the one of Strohman and Yoerger gave the best agreements. The agreement of Ross equation, based on thermodynamics aspects, was tested here for some food sorption isotherms and the agreement was satisfactory.

\section{NOMENCLATURE}

\author{
$\varphi \quad$ relative humidity \\ $X \quad$ moisture content (d.b.) \\ Ps Saturation pressure \\ $\mathrm{s}_{1}$ to $\mathrm{s}_{6}$ Equations parameters
}

$\%$

$$
\begin{aligned}
& \mathrm{kg} \mathrm{kg}^{-1} \\
& \mathrm{kPa}
\end{aligned}
$$

\section{RESUMO}

\section{ISOTERMAS DE SORÇÃO DE ALIMENTOS: ESTUDO DO AJUSTE DE MODELOS MATEMÁTICOS}

O conhecimento das isotermas de sorção é importante para estabelecer as condições de armazenamento de alimentos e de processos como a secagem. Existem diversos modelos de ajuste de isotermas de sorção. Este trabalho apresenta estudo sobre o ajuste de 40 modelos matemáticos de isotermas de sorção para dados experimentais de 53 produtos alimentícios. A soma de resíduo quadrático e o erro padrão foram os critérios de avaliação. Para a maior parte dos produtos, o melhor ajuste foi obtido com a equação de Jaafar e Michalowiski, quando a temperatura e a pressão de saturação não foram consideradas como variáveis. Para os casos em que a temperatura ou a pressão de saturação foram consideradas como variáveis, a equação de Strohman e Yoerger apresentou o melhor ajuste para a maioria dos produtos. A equação de Ross, baseada em aspectos termodinâmicos, também foi testada para alguns produtos, mas o ajuste mostrou-se somente satisfatório.

PALAVRAS-CHAVE: ALIMENTO - ARMAZENAMENTO; SECAGEM; TERMODINÂMICA; ISOTERMAS DE SORÇÃO.

\section{REFERENCES}

1 AFONSO Jr, P.C. Aspectos físicos, fisiológicos e de qualidade do café em função da secagem e do armazenamento. 2001. 384 p. Tese (Doutorado em Engenharia Agrícola), Departamento de Engenharia Agrícola, Universidade Federal de Viçosa, Viçosa, 2001.

2 AGUERRE, R.J.; SUAREZ, C.; VIOLLAZ, P.E. Moisture desorption isotherms of rough rice. International Journal of Food Science and Technology, v. 18, p. 345 - 351, 1983.

3 AJIBOLA, O.O.; AVIARA, N.A.; AJETUMOBI, O.E. Sorption equilibrium and thermodynamic properties of cowpea (Vigna unguiculata). Journal of Food Engineering, v. 58, p. 317-324, 2003.

4 ANDERSON, R.B.; HALL, W.K. Modifications of the Brunauer, Emmett and Teller equation II. Journal of American Chemical Society, v. 70, p. 1727-1734, 1948.

5 ASSUNÇÃO, A.B.; PENA, R.S. Hygroscopic behavior of the dry residue of pink shrimp. Ciência e Tecnologia de Alimentos, v. 27, p. 786-793, 2007.

6 BASU, S.; SHIVHARE, U.S.; MUJUMDAR, A.S. Models for sorption isotherms for foods: a review. Drying Technology, v. 24, p.917-930, 2006. 
BRADLEY, W.M. Polymolecular adsorbed films. Journal of Chemical Society, v. 58, p. 1467-1499, 1936.

8 BROOKER, D.B.; BAKKER-ARKEMA, F.W.; HALL, C.W. Drying cereal grains. Westport: Avi Publishing, 1974. p 70-73.

9 BRUNAUER, S.; EMMETT, P.; TELLER, E. Adsorption of gases in multimolecular layers. Journal of American Chemical Society, v. 60, p. 309-319, 1938.

10 BUSHUK, W.; WINKLER, C.A. Sorption of water vapor on wheat flour, starch and gluten. Cereal Chemistry, v. 34, p. 73-86, 1957.

11 CASTILLO, M.D.; MARTINEZ, E.J.; GONZALEZ, H.H.L.; PACIN, A.M.; RESNIK, S.L. Study of mathematical models applied to sorption isotherms of Argentinean black bean varieties. Journal of Food Engineering, v. 60, p. 343-348, 2003.

12 CAURIE, M. A new model equation for predicting safe storage moisture levels for optimum stability of dehydrated foods. Journal of Food Technology, v.5, p. 301-307, 1970.

13 CHEN, C.S. Equilibrium moisture curves for biological materials. Transactions of the ASAE, v. 14, p. 924-926, 1971.

14 CHEN, C.S.; CLAYTON, J.T. The effect of temperature on sorption isotherms of biological materials. Transactions of the ASAE, v. 14, p. 927-929, 1971.

15 CHUNG, D.S.; PFOST, H. B. Adsorption and desorption of water vapor by cereal grains and their products. Transactions of the ASAE, v. 10, p. 549-557, 1967.

16 COSTA, S.M.; MURATA, V.V.; BARROZO, M.A.S. Drying of soybean seeds in contercurrent and concurrent moving bed. In: INTER-AMERICAN DRYING CONFERENCE, 1997, Itu. Proceedings... Itu: IADC, 1997, p. 561-568.

17 DAY, D.L.; NELSON, G.L. Desorptions isotherms for wheat. Transactions of the ASAE, v. 8, p. 293-297, 1965.

18 DU, S.L.; ZHOU, C. S.; YANG, L.Q. Deacidification of adlay seed (coix lachryma-jobi var. Mayuen) miscella with anionexchange resin. Journal of Food Process Engineering, v. 30, n. 6, p. 729-745, 2007.

19 FABRA, M.J.; TALENS, P.; MORAGA, G., MARTINEZ-NAVARRETE, N. Sorption isotherm and state diagram of grapefruit as a tool to improve product processing and stability. Journal of Food Engineering, v. 93, p. 52-58, 2009.

20 FARAHNAKY, A.; ANSARI, S.; MAJZOOBI, M. Effect of glycerol on the moisture sorption isotherms of figs. Journal of Food Engineering, v. 93, p. 468-473, 2009.

21 GINZBURG, A.S.; SAVINA, I.M. Mass transfer characteristics of food products. Moscow: LiPP, 1982.

22 GOULA, A.M.; KARAPANTSIOS, T.D.; ACHILIAS, D.S.; ADAMOPOULOS, K.G. Water sorption isotherms and glass transition temperature of spray dried tomato pulp. Journal of Food Engineering, v. 85, p. 73-83, 2008.

23 HAILWOOD, A.J.; HORROBIN, S.S. Adsorption of water by polymers: analysis in terms of a simple model. Transactions Faraday Society, v. 42, p. 84-112, 1946.

24 HALSEY, G. Physical adsorption on non-uniform surfaces. Journal of Chemical Physics, v. 16, p. 931-937, 1948.

25 HARKINS, W.D.; JURA, G. Extension of the attractive energy of a solid into an adjacent liquid or film, the decrease of energy with distance, and the thickness of films. Journal of American Chemical Society, v. 66, p. 919-927, 1944a.

26 HARKINS, W.D.; JURA, G. Surfaces of solids. XII. A vapor adsorption method for the determination of the area of a solid without the assumption of a molecular area, and areas occupied by nitrogen and other molecules on the surface of a solid. Journal of American Chemical Society, v. 66, p.1366-1373, 1944b.

27 HAYAKAWA, K.; MATAS, J.; HWANG, M.P. Moisture sorption isotherms of coffee products. Journal of Food Science, v. 43, p. 1026-1027, 1978.

28 HAYNES Jr, B.C. Vapor pressure determination on seed hygroscopicity. Washington, DC: ARS/USDA, 1961. (Technology Bulletin, 1229).

29 HENDERSON, S.M. A basic concept of equilibrium moisture. Agricultural Engineering, v. 33, p. 29- 32, 1952.

30 HUTTIG, G.F. Zur auswertung der adsorptions-isothermen. Monatsh.Chem., v.78, p.177-184, 1948.

31 IGLESIAS, H.A.; CHIRIFE, J. A model for describing the water sorption behaviour of foods. Journal of Food Science, v. 41, p. 984-991, 1976. 
32 IGLESIAS, H.A; CHIRIFE, J. Handbook of food Isotherms. New York: Academic Press, 1982.

33 JAAFAR. F.; MICHALOWSKI, S. Modified bet equation for sorption/desorption isotherms. Drying Technology, v. 8, p. 811-827, 1990

34 KAYMAK-ERTEKIN, F.; GEDIK, A. Sorption isotherms and isosteric heat of sorption for grapes, apricots, apples and potatoes. Lebensmittel- Wissenschaft und-Tecnologie-Food Science \& Technology, v. 37, p. 429-438, 2004

35 KIRANOUDIS, C.T.; TSAMI, E.; MAROULIS, Z.B.; MARINOSKOURIS, D. Drying kinetics of some fruits. Drying Technology, v. 15, p. 1399-1418, 1997.

36 KOC, B.; YILMAZER, M.S.; BALKIR, P.; ERTEKIN, F.K. Moisture sorption isotherms and storage stability of spray-dried yogurt powder. Drying Technology, v. 28, p. 816-822, 2010.

$37 \mathrm{KUHN}, \mathrm{I}$. A new theoretical analysis of adsorption phenomena. Introductory part: the characteristic expression of the main regular types of adsorption isotherms by a single simple equation. Journal Colloid Science, v. 19, p. 685-698, 1964.

38 LANGMUIR, I. The constitution and fundamental properties of solids and liquids. Journal of American Chemical Society, v. 38, p. 2221-2295, 1916.

39 LIMOUSIN, G.; GAUDET, J.P.; CHARLET, L.; SZENKNECT, S.; BARTHE`S, V.; KRIMISSA, M. Sorption isotherms: a review on physical bases, modeling and measurement. Applied Geochemistry, v. 22, p.249-275, 2007.

40 LUZ, G.R.; SOUSA, L.H.C.D.; JORGE L.M.M.; PARAISO, P.R. Study of the equilibrium isotherms of soybean meal. Ciência e Tecnologia de Alimentos, v. 26, p. 408-413, 2006.

41 MADAMBA, P.S.; DRISCOLL, R.H.; BUCKLE, K.A. Models for the specific heat and thermal conductivity of garlic. Drying Technology, v. 13, p. 295-317, 1995.

42 MIZRAHI, S.; LABUZA, T.P.; KAREL, M. Computer-aided predictions of extent of browning in dehydrated cabbage. Journal of Food Science, v. 35, p. 799-803, 1970.

43 MOTARJEMI, Y.A. Study of some physical properties of water in foodstuffs. Water activity, water binding and water diffusivity in minced meat products. 1988. 298 p. Thesis (PhD) - Lund University, Sweden, 1988.

44 OSWIN, C.R. The kinetics of package life III. The isotherm. Journal of Chemical Industry, v. 65, p. 419-421, 1946.

45 PAKOWSKI, Z. DryPak v.1.3: program for psychrometric and drying computations. Lodz: Society of Polish Consultants, 1995.

46 PELEG, M. Assessment of a semi-empirical four parameter general model for sigmoid moisture sorption isotherms. Journal of Food Processing Engineering, v. 16, p. 21-37, 1993.

47 POLLIO, M.L.; TOLABA, M.P.; SUAREZ, C. Measuring and modeling grain sorption equilibria of amaranth grains. Cereal Chemistry, v. 75, p. 297-300, 1988.

48 ROMAN, A. D.; HERMAN-Y-LARA, E.; SALGADO-CERVANTES, M.A.; GARCIA-ALVARADO, M.A. Food sorption isotherms prediction using the Ross equation. Drying Technology, v. 22, p.1829-1843, 2004.

49 ROUNSLEY, R.R. Multimolecular adsorption equation. A.I.Ch.E. Journal, v. 7, p. 308-311, 1961.

50 SHIVHAREA, U.S.; ARORAB, S.; AHMEDC, J.; RAGHAVAN, G.S.V. Moisture adsorption isotherms for mushroom. Lebensmittel- Wissenschaft Und-Tecnologie-Food Science \& Technology, v. 37, p. 133-137, 2004.

51 SHOTTON, E.; HARB, N. The effect of humidity and temperature on the equilibrium moisture content of powders. Journal of Pharmacy and Pharmacology, v. 17, p. 504-508, 1965.

52 SILVA, A.E.; SILVA, L.H.M.; PENA, R.S. Comportamento higroscópico do açaí e cupuaçu em pó. Ciência e Tecnologia de Alimentos, v. 28, p. 895-901, 2008.

53 SILVA, R.G.; ALMEIDA, C.A.A.; SILVA, M.M.; GOUVEIA, J.P.G.; ALMEIDA, F.A.C.; SILVA, F.L.H. Comportamento das isotermas de umidade em frutos de acerola. In: SIMPÓSIO BRASILEIRO DE PÓS COLHEITA DE FRUTOS TROPICAIS, 2005, João Pessoa. Anais... João Pessoa: SBPCFT, 2005.

54 STROHMAN, R.D.; YOERGER, R.R. A new equilibrium moisture content equation. Transactions of the ASAE, v.10, p. 675-677, 1967.

55 SYAMALADEVI, R.M.; SABLANI, S.S.; TANG, J.M.; POWERS, J.; SWANSON, B.G. Water sorption and glass transition temperatures in red raspberry (Rubus idaeus). Thermochimica Acta, v. 503, p. 90-96, 2010 
56 THYS, R.C.S.; NORENA, C.P.Z.; MARCZAK, L.D.F.; AIRES, A.G.; CLARERA-OLIVEIRA, F. Adsorption isotherms of pinhao (Araucaria angustifolia seeds) starch and thermodynamic analysis. Journal of Food Engineering, v. 100, p. 468-473, 2010.

57 TOLABA, M.P.; MERCEDES, P.; ENRIQUEZ, N.; POLLIO, M. A. Grain sorption equilibria of quinoa grains. Journal of Food Engineering, v. 61, p. 365-371, 2004.

58 TRUJILLO, F.J.; YEOW, P.C.; PHAM, Q.T. Moisture sorption isotherm of fresh lean beef and external beef fat. Journal of Food Engineering, v. 60, p. 357-366, 2003.

59 VAN DEN BERG, C. Description of water activity of foods for engineering purposes by means of the GAB model of sorption. In: MCKENNA, B.M. (Ed.). Engineering and food. London: Elsevier Applied Science, 1984. p.311-321.

60 VASCONCELOS, L.H. Determinação das propriedades físicas de canola (Brassica napus) Variedade Iciola 41, relacionadas a armazenagem. 1998. 220 p. Dissertação (Mestrado em Engenharia Agrícola) Faculdade de Engenharia Agrícola, Universidade Estadual de Campinas, Campinas, 1998.

61 VEgA-GÁlVEZ, A.; LeMUS-MONDACA, R.; TELLO-IRELAND, C.; MIRANDA, M.; YAGNAM, F. Kinetics study of convective drying of blueberry variety O'NEIL (Vaccinium corymbosum L.). Chilean Journal of Agricultural Research, v. 69, p. 171-178, 2009.

62 WERLING, K. Adiabatic drying of hygroscopic materials. In: DRYING SYMPOSIUM, 1978, Montreal. Proceedings... Montreal: DS, 1978. p. 36.

63 YANG, B; GAO, Y.X.; LIU, X.A.; LI, Y.Y.; ZHAO, J. Adsorption characteristics of crocin in the extract of gardenia fruits jian (gardenia jasminoides ellis) on macroporous resins. Journal of Food Process Engineering, v. 32, n. 1, p. 35-52, 2009.

\section{ACKNOWLEDGEMENTS}

The authors thank Profa. Dra. Maria Aparecida Silva for the suggestion of the theme of this work. 